\title{
FREDHOLM REALIZATIONS OF ELLIPTIC SYMBOLS ON MANIFOLDS WITH BOUNDARY
}

\author{
PIERRE ALBIN AND RICHARD MELROSE
}

\begin{abstract}
We show that the existence of a Fredholm element of the zero calculus of pseudodifferential operators on a compact manifold with boundary with a given elliptic symbol is determined, up to stability, by the vanishing of the Atiyah-Bott obstruction. It follows that, up to small deformations and stability, the same symbols have Fredholm realizations in the zero calculus, in the scattering calculus and in the transmission calculus of Boutet de Monvel.
\end{abstract}

\section{Contents}

Introduction

1. Operators

1.1. Zero calculus

1.2. Model zero structure

1.3. Reduced normal operator, order $-\infty$

1.4. Reduced normal operator, general order

2. K-theory

2.1. Smooth K-theory

2.2. K-theory sequence

2.3. $\quad C^{*}$ K-theory

3. Relation between b and cusp calculi

4. Fredholm perturbations of the identity 16

5. Six term exact sequence 18

6. Relation to boundary value problems 21

6.1. Atiyah-Patodi-Singer versus local boundary conditions 21

6.2. Atiyah-Bott obstruction 22

6.3. Boutet de Monvel transmission algebra 23

6.4. Relationship to scattering and zero calculi 24

\begin{tabular}{|l|l|} 
6.5. Differential operators & 25 \\
\hline
\end{tabular}

$\begin{array}{ll}\text { References } & 27\end{array}$

The first author was partially supported by an NSF postdoctoral fellowship and the second author received partial support under NSF grant DMS-0408993. 


\section{INTRODUCTION}

Consider a compact manifold with boundary, $X$, or more generally a fibration

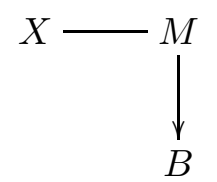

with typical fibre $X$. To a symbol

$$
a \in \mathcal{C}^{\infty}\left(S^{*}(M / B) ; \operatorname{hom}(E, F)\right)
$$

for vector bundles $E, F$ over $M$, one can associate, after a little 'preparation' various choices of families of pseudodifferential operators with symbol $a$. In particular, we may consider operators of 'zero' type in $\Psi_{0}^{0}(M / B ; E, F)$ or of 'scattering' type in $\Psi_{\mathrm{sc}}^{0}(M / B ; E, F)$, or of 'transmission' type in Boutet de Monvel's algebra $\Psi_{\operatorname{Tr}}^{0}(M / B ; E, F)$. In these, and other, cases we can seek the conditions on $a$ under which it has a realization, in the corresponding sense, which is Fredholm on the natural $L^{2}$-spaces. The primary condition is always symbolic ellipticity, that $a^{-1} \in \mathcal{C}^{\infty}\left(S^{*}(M / B)\right.$; hom $\left.(F, E)\right)$ should exist. For the three 'quantization procedures' mentioned above such a Fredholm realization of an elliptic symbol exists if and only if the K-class associated to the symbol, in terms of absolute K-theory with compact supports, lies in the image of the K-theory supported in the interior

$$
\mathrm{K}_{\mathrm{c}}\left(T^{*}(\operatorname{int}(M) / B)\right) \ni \alpha \mapsto i_{*}(\alpha)=[a] \in \mathrm{K}_{\mathrm{c}}\left(T^{*}(M / B)\right) .
$$

This is established here for the zero calculus (as introduced in [15], [17]) by computing K-theory invariants of the algebra. For the scattering calculus it is shown in [26] and for the transmission case already in essence by Boutet de Monvel in [6] (see the much more recent work by Melo, Nest, and Schrohe [19] and Melo, Schick, and Schrohe [20]); this in turn is an extension of the results of Atiyah and Bott [3].

The elements of the Lie algebra, $\mathcal{V}(X)$, of smooth vector fields on a compact manifold with boundary act on $\mathcal{C}^{\infty}(X)$. If $0<\nu \in \mathcal{C}^{\infty}(X ; \Omega)$ is a smooth density and $d(V) \in \mathcal{C}^{\infty}(X)$ is the divergence of $V$ with respect to $\nu$ then

$$
\int_{X}(V u) \cdot w \nu=\int_{X} u \cdot(-V+d(V) w) \nu+\int_{\partial X} n_{V} u \cdot w \nu^{\prime}
$$

where $n_{v} \in \mathcal{C}^{\infty}(\partial X)$ is the normal component of $V$ at $\partial Z$ computed with respect to a density $0<\nu^{\prime} \in \mathcal{C}^{\infty}(\partial X ; \Omega)$. Thus, in order to get an action of the associated enveloping algebra, one needs either to add boundary conditions, as in the transmission algebra, or restrict to elements $V \in \mathcal{V}_{\mathrm{b}}(X) \subset \mathcal{V}(X)$ of the Lie algebra of vector fields tangent to the boundary for which $n_{V}=0$.

Associated to $\mathcal{V}_{\mathrm{b}}$ is an algebra of pseudodifferential operators $\Psi_{\mathrm{b}}^{\mathbb{Z}}(X)$ containing the enveloping algebra $\operatorname{Diff}_{\mathrm{b}}^{\mathbb{N}}(X) \supset \mathcal{V}_{\mathrm{b}}(X)$ and acting continuously, 
with adjoints, on $\mathcal{C}^{\infty}(X)$. The same is true for various Lie subalgebras of $\mathcal{V}_{\mathrm{b}}(X)$. Here we are most interested in the natural ones:

$$
\begin{gathered}
\mathcal{V}_{0}(X)=\left\{V \in \mathcal{V}_{\mathrm{b}}(X): V=x W, W \in \mathcal{V}(X)\right\} \\
\mathcal{V}_{\mathrm{sc}}(X)=\left\{V \in \mathcal{V}_{\mathrm{b}}(X): V=x W, W \in \mathcal{V}_{\mathrm{b}}(X)\right\}
\end{gathered}
$$

fixed in terms of a boundary defining function $x \in \mathcal{C}^{\infty}(X)$ on which they do not depend. There are other interesting algebras which depend on some choice of, or the existence of, more structure. These include

$$
\begin{aligned}
\mathcal{V}_{\mathrm{cu}}(X) & =\left\{V \in \mathcal{V}_{\mathrm{b}}(X): V \cdot x \in x^{2} \mathcal{C}^{\infty}(X)\right\} \\
\mathcal{V}_{\psi-\mathrm{b}}(X) & =\left\{V \in \mathcal{V}_{\mathrm{b}}(X): V \cdot \mathcal{C}_{\psi}^{\infty}(X) \subset x \mathcal{C}^{\infty}(X)\right\} \\
\mathcal{V}_{\psi-\mathrm{cu}}(X) & =\mathcal{V}_{\mathrm{cu}}(X) \cap \mathcal{V}_{\psi-\mathrm{b}}(X) \\
\mathcal{V}_{\theta}(X) & =\left\{V \in \mathcal{V}_{0}(X): V \cdot \widetilde{\theta} \in x^{2} \mathcal{C}^{\infty}(X)\right\}
\end{aligned}
$$

where $\partial X \stackrel{\psi}{\rightarrow} Y$ is a fibration of the boundary, $x \in \mathcal{C}^{\infty}(X)$ is a boundary defining function (on which for instance $\mathcal{V}_{\text {cu }}$ depends) and $\widetilde{\theta} \in \mathcal{C}^{\infty}\left(X, \Lambda^{1}\right)$ is a real 1 -form with $\theta=i_{\partial X}^{*} \widetilde{\theta}$ non-vanishing.

In all of these cases there is a well-defined algebra of pseudodifferential operators, denoted $\Psi_{S}^{\mathbb{Z}}(X)$, for the 'structures' $S=\mathrm{b}, 0$, sc, cu, $\psi$-b, $\psi$-cu, $\theta$ which includes the corresponding enveloping algebra.

Since the operators of order zero act on $\mathcal{C}^{\infty}(X)$ as a $*$-algebra and extend to bounded operators on $L^{2}(X)$ the $C^{*}$-closure exists

$$
\overline{\Psi_{S}^{0}} \subset \mathcal{B}\left(L^{2}(X)\right),
$$

so the K-theory of this, and related algebras, is defined. In all cases

$$
\dot{\Psi}^{-\infty}(X) \equiv \dot{C}^{\infty}\left(X^{2} ; \Omega_{R}\right),
$$

the space of smoothing operators with kernels vanishing to infinite order at the boundary, is an ideal. It has closure $\mathfrak{K}$, the ideal of compact operators and so leads to the sequence

$$
\mathfrak{K} \longrightarrow \overline{\Psi_{S}^{0}}(X) \longrightarrow \mathcal{A}_{S}^{0}(X)=\overline{\Psi_{S}^{0}}(X) / \mathfrak{K}
$$

and the associated 6-term exact 'index sequence' in K-theory.

It is also possible to construct a smooth version of K-theory, without passing to the $C^{*}$-closure, giving however essentially the same result (see 2.3 ). In some cases this is a little delicate since these algebras may not admit the functional calculus, in particular this is the case for the 'regular singular' algebras corresponding to $S=\mathrm{b}, 0, \psi$-b, $\theta$. Nevertheless we may define a 'geometric' replacement for $K_{1}\left(\mathcal{A}_{S}^{0}\right)$ as in [26], which we denote $\mathcal{K}_{S}^{0}(X)$ and more generally $\mathcal{K}_{S}^{0}(M / B)$ in the case of a fibration (11) and, as necessary, possessing fibrewise structure $S$. This K-group is identified as the stable homotopy classes, up to bundle isomorphism, of the elliptic and Fredholm elements of the modules $\Psi_{S}^{0}(M / B ; E, F)$ acting between sections of vector 
bundles. The analytic index for families then becomes a homomorphism

$$
\mathcal{K}_{S}^{0}(M / B) \stackrel{\operatorname{ind}_{a}}{\longrightarrow} K(B) .
$$

Again in all the cases listed above (and others) there is a 'symbolically trivial' ideal

$$
\Psi_{S}^{-\infty}(X) \subset \Psi_{S}^{0}(X)
$$

which contains non-compact operators, and a corresponding smooth $\mathrm{K}$ group, $\mathcal{K}_{-\infty, S}^{0}(M / B)$, corresponding to Fredholm operators of the form $\mathrm{Id}+A, A \in \Psi_{S}^{-\infty}(X)$. The short exact sequence of algebras

$$
\Psi_{S}^{-\infty}(X) \stackrel{I}{\longrightarrow} \Psi_{S}^{0}(X) \longrightarrow \mathcal{C}^{\infty}\left({ }^{S} S^{*} X\right)[[\rho]]
$$

with image the star algebra for symbols in terms of a quantization map, induces a 6 -term exact sequence in the smooth K-theory

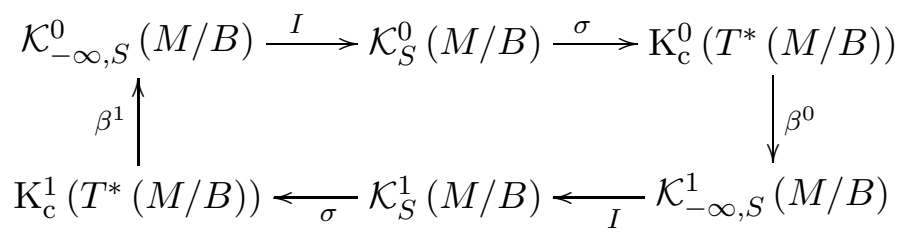

although it should be emphasized that we do not construct a general theory from which this follows.

In terms of K-theory, the algebras again determine two extreme cases which we call the universal (for symbols) case and the geometric case, with all others 'intermediate'. The universal cases are characterized by the fact that the 6-term exact sequence above decouples into two short exact sequences

$$
S \text { universal } \Longleftrightarrow \mathcal{K}_{-\infty, S}^{i}(M / B)=\mathrm{K}_{\mathrm{c}}^{i}(B \times \mathbb{R}), \beta^{0}=\beta^{1}=0 .
$$

Theorem 1 ([26], see Corollary 3.2 below). The cusp and $b$ structures are universal.

The second extreme case is characterized by

$S$ is geometric $\Longleftrightarrow$

the 6 -term exact sequence (3) reduces to the geometric sequence:

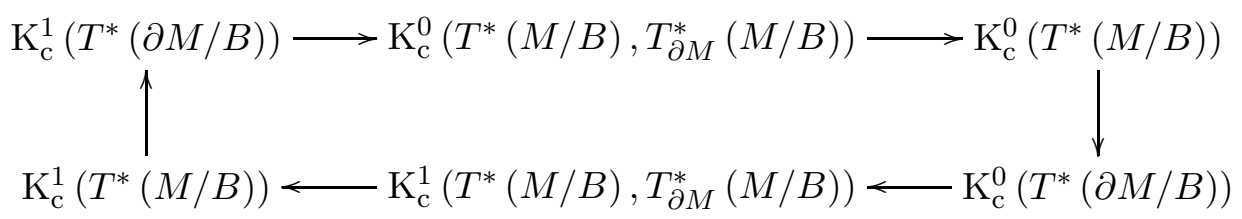

in which the connecting homomorphisms are geometric boundary maps (and the K-theory of manifolds with boundary is absolute).

Theorem 2 ([26] for sc, Proposition 5.3 below for zero). The structures 0 and $\mathrm{sc}$ are geometric. 
This is the main technical result of this paper, it answers the question posed by the title for the zero calculus in the form:

Proposition 3. An elliptic symbol $a \in \mathcal{C}^{\infty}\left(S^{*}(M / B) ;\right.$ hom $\left.(E, F)\right)$ has a realization as the symbol of a Fredholm element of $\Psi_{0}^{0}(M / B ; E, F)$ if and only if for some $N$ there exists

$$
\begin{aligned}
\widetilde{a} \in \mathcal{C}^{\infty}\left(\overline{T^{*} \partial M / B} ; \operatorname{hom}(\right. & \left.\left.E \oplus \mathbb{C}^{N}, F \oplus \mathbb{C}^{N}\right)\right) \text { with } \\
& \widetilde{a}^{-1} \in \mathcal{C}^{\infty}\left(\overline{T^{*} \partial M / B} ; \operatorname{hom}\left(F \oplus \mathbb{C}^{N}, E \oplus \mathbb{C}^{N}\right)\right)
\end{aligned}
$$

such that

$$
a \oplus \operatorname{Id}_{\mathbb{C}^{N}} L_{S^{*} \partial M / B}=\widetilde{a} L_{S^{*} \partial M / B} .
$$

That is, a can be (stably) trivialized over the boundary.

In the universal case as opposed to the geometric cases there is no restriction on the symbol other than invertibility (hence the name).

The 'intermediate' case $S=\psi$-cu for $\psi$ a non-trivial fibration of the boundary (so both fibre and base having positive dimension) is discussed in [26]. Using an 'adiabatic limit' construction it will be shown in a subsequent paper that there are natural isomorphisms

$$
\mathcal{K}_{\psi \text {-cu }}^{i}(M / B) \longleftrightarrow \mathcal{K}_{\psi \text {-b }}^{i}(M / B) \longleftrightarrow K K_{B}^{i}\left(C_{\psi}(M), C(B)\right)
$$

where the KK-functor (linear over $K(B)$ ) is applied to the continuous functions on $M$ which are fibre constant for $\psi$. The boundary maps for the 6 -term sequence then reduce to versions of the Atiyah-Singer index map for the fibration of the boundary.

Before detailing the various sections of this paper, we mention some related literature. The article [7] was released shortly after the first version of this article and continues previous work of Lauter [12] regarding the $C^{*}$ algebras generated by operators in the zero calculus on a single manifold. In it, Ditsche computes the K-theory of the corresponding Calkin algebra. We show that this computation is equivalent to ours in $\$ 2.3$ in the case of a single manifold, though for a family of manifolds one would have to consider a KK group as explained in [26].

The approach of Savin in [28] deals with manifolds with non-trivial fibrations at the boundary. He shows that groups of elliptic operators, similar to those we study here, are isomorphic to the K-homology of the stratified manifold resulting from collapsing the fibration. In the geometric case treated here, Savin recovers the classical Atiyah-Bott obstruction as the obstruction to the existence of elliptic boundary conditions of Lopatinskii-Schapiro type.

Finally, we also mention [29] where an extension of the transmission algebra is decribed so as to allow arbitrary elliptic operators to be quantized as Fredholm operators. This approach is extended in 30 to remove the transmission condition on the principal symbol and in [31] to allow for a non-trivial fibration at the boundary. 
After briefly recalling the properties of the zero calculus in $\$ 1$ smooth K-theory is defined in 92 , and in $\$ 3$ we discuss the relation between the b and cusp calculi, both to review the universality, in the sense discussed above, of their K-theory and to establish the closely related issue of the (weak) contractibility of certain groups and semigroups. Then, in this contractibility is used to identify $\mathcal{K}_{-\infty, 0}^{0}(M / B)$. The six term exact sequence and the identification of the group $\mathcal{K}_{0}^{0}(M / B)$ is carried out in 95 . In the final section the approach of Boutet de Monvel is reviewed and compared to the scattering and zero calculi. The analogue of Proposition 3 holds for the transmission algebra, and corresponds to the obstruction to the existence of local boundary conditions found by Atiyah and Bott, and we show that the various lifts to $K^{0}\left(T^{*} M / B, T_{\partial M}^{*} M / B\right)$ are consistent, i.e., they yield the same index.

We are happy to acknowledge the helpful comments of the referee of this paper.

\section{Operators}

1.1. Zero calculus. The zero calculus was defined and developed in [15], [17, [16], [12]. In this section we briefly recall the definitions and particularly those facts about the reduced normal operator that we need below.

The space of 0 -vector fields is defined on any manifold with boundary by (2). In local coordinates, $x, z_{i}$ where $x$ is a boundary defining function, and $z_{i}$ are coordinates along the boundary, $\mathcal{V}_{0}$ is locally spanned by $x \partial_{x}, x \partial_{z_{i}}$. Zero differential operators are elements of the enveloping algebra of $\mathcal{V}_{0}$ and therefore are locally of the form

$$
\sum_{j+|\alpha| \leq k} a_{j, \alpha}(x, z)\left(x \partial_{x}\right)^{j}\left(x \partial_{z}\right)^{\alpha}
$$

with arbitrary smooth coefficients. Such differential operators are contained in the algebra of zero pseudodifferential operators. In the references above the latter are identified as a space of conormal distributions on a suitable modification of the double space $X^{2}$ (the zero stretched double space $X_{0}^{2}$ ).

The space of zero pseudodifferential operators of order $k$ acting between sections of the vector bundles $E$ and $F$ is denoted $\Psi_{0}^{k}(X ; E, F)$. There are two model operators. The first is the principal symbol which corresponds to the short exact sequence

$$
\Psi_{0}^{k-1}(X ; E, F) \hookrightarrow \Psi_{0}^{k}(X ; E, F) \stackrel{\sigma}{\rightarrow} \mathcal{C}^{\infty}\left(S^{*} X ; \operatorname{hom}\left(\pi^{*} E, \pi^{*} F\right) \otimes N_{k}\right)
$$

The second is the normal operator which models the behavior at the boundary and corresponds to a short exact sequence

$$
x \Psi_{0}^{k}(X ; E, F) \hookrightarrow \Psi_{0}^{k}(X ; E, F) \stackrel{N}{\rightarrow} \Psi_{\mathrm{inv}}^{k}(\widetilde{G} / \partial X ; E, F) .
$$

Here, $\widetilde{G} \longrightarrow \partial X$ is a fibration of compact manifolds which arises geometrically as the front face of the stretched double space; it is discussed more 
extensively below. The fibres are closed quarter spheres which combine to give a compactification of the bundle of (solvable) Lie groups over $\partial X$ corresponding to the (canonical) 0-structure. The range space in (1.1), which we characterize in more detail below, consists of distributional sections of a bundle over $\widetilde{G}$ which are smooth away from the identity section, where they have a classical conormal singularity, and which vanish to infinite order at the boundaries. The product is just the smooth family of convolution products, on the fibres, with 'inv' standing for this invariant, i.e. convolution, product.

These two symbol homomorphisms, taken together, are analogous to the principal symbol in the pseudodifferential calculus on a closed manifold. Thus, for instance, a pseudodifferential operator acting on the appropriate $L^{2}$-space is compact precisely when both symbols vanish; it is Fredholm when both symbols are invertible. In the case of the convolution algebra on the solvable group, invertibility is in the sense of bounded operators on the natural Sobolev space and the inverse need not be of the same form. In preparation for other formulations of the normal operator we reinterpret the naturality of the zero structure and describe alternative constructions of the bundle of groups and $\Psi_{\text {inv }}$.

1.2. Model zero structure. Let $V$ be a real vector space of dimension $n$ and suppose that $L^{+} \subset V^{\prime}$ is a half-line in the dual, so

$$
\exists v^{\prime} \in V^{\prime} \text { s.t. } w^{\prime} \in L^{+} \Longleftrightarrow w^{\prime}=t v^{\prime} \text { for } t>0 .
$$

With $V$ we may associate another vector space of the same dimension

$$
\widetilde{V}=L \otimes V, L=\mathbb{R} \cdot L^{+} .
$$

Thus, $\widetilde{V}$ can be identified as the space of linear vector fields on $V$ (sums of products of linear functions and constant vector fields) where the linear coefficients are in $L$. As such it is a solvable Lie algebra, more precisely if we choose a decomposition of $V=\mathbb{R}_{x} \times \mathbb{R}_{y}^{n-1}$ in which $L^{+}$is spanned by $x$, the dual of the first factor, then $\widetilde{V}$ is spanned by

$$
x \partial_{x}, x \partial_{y_{i}} \text {. }
$$

The span of the second class of vector fields is a subspace of dimension $n-1$ which is well-defined as the center of the Lie algebra

$$
\widetilde{W} \subset \widetilde{V} \text {. }
$$

The elements of $\widetilde{V}$ are clearly complete as vector fields on $V$ so choosing a point $p \in V^{+}$, i.e. where $L^{+} \geq 0$, then $V^{+}$has a Lie group structure with identity $p$ and Lie algebra $\widetilde{V}$ and this is the unique connected and simply connected group (up to isomorphism) with this Lie algebra. The centre $\widetilde{W}$ integrates to a foliation of $V^{+}$over $(0, \infty)$ which has an induced multiplicative structure coming from the short exact sequence of Lie groups

$$
L^{\circ} \longrightarrow G_{V, L^{+}} \longrightarrow(0, \infty)
$$


i.e. $G_{V, L^{+}}$is a semidirect product. Choosing a 'normal element' in $\widetilde{V}$, complementary to $\widetilde{W}$ and acting on it as the identity through the Lie bracket, fixes an image of $(0, \infty)$ in $G_{V, L^{+}}$and hence identifies all the fibres with $\widetilde{W}$ as a vector space (rather than just an affine space). The compactification of $G_{V, L^{+}}$to $\widetilde{G}_{V, L^{+}}$, simply the radial compactification of the closure in $V$, gives a compact manifold independent of choices, since linear transformations preserving the boundary lift to be smooth on the compactification.

Applying these constructions to fibres of the tangent bundle to $X$ over the boundary, with $L^{+}$being the positive part of the conormal line, gives a bundle of groups over $\partial X$. Note that the front face of the zero double space has interior isomorphic to the inward-point half of the tangent bundle to the boundary, with a canonical interior 'identity' section. Namely, the interior is canonically the inward-pointing part of the (projective) spherical normal bundle to the boundary of the diagonal. This consists of vectors $\left(v_{R}, v_{L}\right)$, lifted from left and right, modulo those tangent to the boundary of the diagonal - which are of the form $\left(v^{\prime}, v^{\prime}\right)$ where $v^{\prime}$ is tangent to the boundary - and modulo the $\mathbb{R}^{+}$action. It follows that if $N$ is inward pointing then $(N, N)$ is a well-defined interior point of the front face. For this reason the bundle of group structures on the front face is well-defined. Note that to extend this natural identity point to an origin for each of the tangential fibres it is only necessary to choose a global inward-pointing vector field $N$ since then the points $(t N, N), t>0$, become origins of the abelian fibres.

1.3. Reduced normal operator, order $-\infty$. To understand the structure of the normal operator better we use the Fourier decomposition corresponding to the abelian fibres of the solvable Lie group to 'reduce' it to a parametrized family of operators on an interval. In the case of operators of order $-\infty$ this was carried out explicitly by Lauter, [12], who characterized the range of the homomorphism to this reduced normal operator. The normal operator in (1.1) at each fibre takes values in $\dot{\mathcal{S}}\left([0, \infty)_{s} \times \mathbb{R}_{Y}^{n-1}\right)$, the space of Schwartz functions on $\mathbb{R}^{n}$ with support in this half-space. The reduced normal operator corresponds to Fourier transformation in $Y$, followed by the introduction of polar coordinates in the dual variable. The result

$$
\mathcal{N}(s,|\eta|, \widehat{\eta}) \in \mathcal{S}\left([0, \infty)_{s} \times[0, \infty) \times \mathbb{S}_{\widehat{\eta}}^{n-2}\right)
$$

vanishes to infinite order at $s=0$ and may be interpreted as the kernel of an operator on the $|\eta|$ half-line. Namely we may identify $(0, \infty) \times(0, \infty)$ with the interior of

$$
I_{\mathrm{b}, 0}^{2}=\left[I^{2} ;\{(0,0)\}\right], I=[0,1]
$$

by the map

$$
(s, r) \longrightarrow(s r, r) \in(0, \infty)^{2} \longrightarrow\left(\frac{s r}{1+s r}, \frac{r}{1+r}\right) \in I^{2} .
$$

In terms of the variables $s,|\eta|, \widehat{\eta}$ in (1.7), $\mathcal{N}(s,|\eta|, \widehat{\eta})$ is an arbitrary smooth function which decays rapidly with all derivatives as $s \rightarrow 0, s \rightarrow \infty$ 
and $|\eta| \rightarrow \infty$, except that there is special behaviour at $|\eta|=0$ where it is, of course, a smooth function of $\eta$ not just smooth in $|\eta|$ and $\widehat{\eta}$.

Let $\pi$ be the projection $S^{*} \partial X \longrightarrow \partial X$, let $(y, \eta)$ denote a point in $S^{*} \partial X$, and let $\left(\tau, \rho_{b}\right) \in[-1,1] \times \mathbb{R}^{+}$be coordinates in the $\mathrm{b}$, cu-double space $\left(\rho_{b}=0\right.$ defines the b-front face).

Proposition 1.1 ( $c f$ [12], Prop. 4.4.1). The reduced normal operator defines an isomorphism of algebras

$$
\Psi_{\text {inv }}^{-\infty}(\widetilde{G} / \partial X ; E) \longleftrightarrow \operatorname{Ran}^{-\infty}(\mathcal{N}) \subset \Psi_{\text {b,cu }}^{-\infty, \cdot}\left(\mathcal{I} / S^{*} \partial X ; E\right)
$$

where $\Psi_{\mathrm{b}, \mathrm{cu}}^{-\infty} \cdot\left(\mathcal{I} / S^{*} \partial X ; E\right)$ is the space of b-pseudodifferential operators of order $-\infty$, on the compactified radial interval bundle, vanishing rapidly at the 'infinite' (cusp) end and $\operatorname{Ran}^{-\infty}(\mathcal{N})$ is the subspace with the additional constraint that the Taylor series at the front face is of the form

$$
\sum_{\alpha} I_{\alpha}(s) \eta^{\alpha}
$$

1.4. Reduced normal operator, general order. In the general case of operators of integral order, the reduced normal operator is defined in the same way but takes values in the pseudodifferential operators on the unit interval which localize to an element of the b-calculus near the left endpoint and to an element of the (weighted) cusp calculus near the right endpoint with parameters in the cosphere bundle of the boundary. Indeed, if $A \in$ $\Psi_{0}^{k}(X)$, then near the boundary the kernel of $A$ is given locally by

$$
\mathcal{K}_{A}(\tau, U, r, y)=\int_{\mathbb{R}_{\eta}^{n-1} \times \mathbb{R}_{\xi}} e^{i(U \cdot \eta+\tau \xi)} a(r, y, \xi, \eta) d \xi d \eta
$$

for a symbol $a \in S_{c l}^{m}\left(\mathbb{R}_{+} \times \mathbb{R}_{y}^{n-1} ; \mathbb{R}_{\xi} \times \mathbb{R}_{\eta}^{n-1}\right)$. We obtain the reduced normal operator by restricting to the zero front face $(r=0)$ and Fourier transforming in the horizontal directions. The kernel of the reduced normal operator (as a half-density) is given by

$$
\mathcal{K}_{\mathcal{N}(A)}\left(y, \eta ; \tau, \rho_{b}\right)=\int_{\mathbb{R}_{\xi}} e^{i \tau \xi} a\left(0, y, \xi, \rho_{b} \eta\right) d \xi\left|\frac{d \rho_{b}}{\rho_{b}} d \tau\right|^{\frac{1}{2}} .
$$

This kernel defines, for fixed $(y, \eta)$, a b,c-operator on the interval $\mathcal{I}=[0,1]$.

This family of b,c-operators has, in turn, three model operators, the interior symbol corresponding to the conormal singularity at the diagonal, an indicial family at the b-end, and a suspended family at the cusp end (see [21], [18] respectively). The interior symbol is given at the point $(u ; \omega) \in S^{*} \mathcal{I}$ $(=\mathcal{I} \times\{ \pm 1\})$ by

$$
{ }^{b, c} \sigma(\mathcal{N}(A)(y, \eta))(u, \omega)={ }^{0} \sigma(A)(0, y ; \omega, 0)
$$

as can be checked from (1.12) using the Taylor expansion of $a(r, y, \xi, \eta)$ in $\eta$. The kernel of the operator restricted to the b-face is

$$
\int_{\mathbb{R}_{\xi}} e^{i \tau \xi} a(0, y, \xi, 0) d \xi
$$


and Mellin transform in $\tau$ produces the b-indicial family.

For the behavior near the cusp end, we first introduce the new coordinate $\rho_{\mathrm{cu}}=\frac{1}{\rho_{\mathrm{b}}}$. The operator is then defined for $\left(\tau, \rho_{\mathrm{cu}}\right) \in[-1,1] \times[0,1]$, and the cusp front face is obtained by blowing up the point $(0,0)$. Lifting $\mathcal{N}(A)$ to the blown-up space (introducing $T=\frac{\tau}{\rho_{\mathrm{cu}}}$ ) and restricting to a neighborhood near the new front face and the diagonal, the kernel takes the form

$$
\begin{gathered}
\mathcal{K}_{\mathcal{N}(A)(y, \eta)}(s, T)=\int_{\mathbb{R}_{\xi}} e^{i \rho_{\mathrm{cu}} T \xi} a\left(0, y, \xi, \frac{\eta}{\rho_{\mathrm{cu}}}\right) d \xi \rho_{\mathrm{cu}}\left|\frac{d \rho_{\mathrm{cu}}}{\rho_{\mathrm{cu}}^{2}} d T\right|^{\frac{1}{2}} \\
\stackrel{\widehat{\xi}=\rho_{\mathrm{cu} \xi}}{\longrightarrow} \int_{\mathbb{R}_{\xi}} e^{i T \widehat{\xi}} a\left(0, y, \frac{\widehat{\xi}}{\rho_{\mathrm{cu}}}, \frac{\eta}{\rho_{\mathrm{cu}}}\right) d \widehat{\xi}\left|\frac{d \rho_{\mathrm{cu}}}{\rho_{\mathrm{cu}}^{2}} d T\right|^{\frac{1}{2}} .
\end{gathered}
$$

Thus the restriction to the cusp front face has kernel

$$
\rho_{\mathrm{cu}}^{-k} \int_{\mathbb{R}_{\xi}} e^{i T \widehat{\xi}}{ }^{0} \sigma(A)(0, y, \widehat{\xi}, \eta) d \widehat{\xi}
$$

and the cusp suspended family is given by

$$
I_{\mathrm{cu}}(\mathcal{N}(A)(y, \eta))(\xi)={ }^{0} \sigma(A)(0, y, \xi, \eta) .
$$

In this way,

$$
\mathcal{N}: \Psi_{0}^{k}(X ; E, F) \longrightarrow \rho_{\mathrm{cu}}^{-k} \Psi_{\mathrm{b}, \mathrm{cu}}^{k}\left(\mathcal{I} / S^{*} \partial X ; E, F\right) .
$$

These operators act on the fibres of the radial part of the dual spaces to the abelian fibres in the compactified group. As in the smoothing case a key feature of the reduced normal operator is that the b-indicial family only depends on the parameters in $\partial X$ and not on the fibre in $S^{*} \partial X$. Although we do not give an explicit characterization of the range of (1.14) the following exact sequence suffices for our purposes.

Proposition 1.2. The (full) b-symbol map on the range $\operatorname{Ran}^{k}(\mathcal{N})$ of the reduced normal operator in (1.14) leads to a short exact sequence (with $k^{\prime}=$ $\left.k+\frac{n}{4}\right)$

$$
\rho_{b}^{\infty} \rho_{\mathrm{cu}}^{-k} \Psi_{\mathrm{b}, \mathrm{cu}}^{-\infty}\left(\mathcal{I} / S^{*} \partial X ; E, F\right) \hookrightarrow \operatorname{Ran}^{k}(\mathcal{N}) \rightarrow I_{\mathcal{S}}^{k^{\prime}}\left(\mathcal{I}_{\partial X}\right)[[\eta]],
$$

where the image space consists of the formal power series in $\eta$ with the coefficient of $\eta^{\alpha}$ being an arbitrary element of the space $I_{\mathcal{S}}^{k^{\prime}-|\alpha|}\left(\mathcal{I}_{\partial X}\right)$ of conormal distributions which are Schwartz at infinity and the null space is precisely the space of (b-)cusp operators of symbolic order $-\infty$, vanishing to infinite order at the ' $b$-end' and of singularity order $k$ at the cusp end (and in this sense arbitrary smooth sections of the bundle over $\left.S^{*} \partial X\right)$. 
Proof. From the discussion above, the full b-indicial operator is given by the Taylor expansion of the kernel at $\rho_{b}=0$ :

$$
\begin{aligned}
\mathcal{K}_{\mathcal{N}(A)}\left(y, \widehat{\eta} ; \tau, \rho_{b}\right) & =\int_{\mathbb{R}_{\xi}} e^{i \tau \xi} a\left(0, y, \xi, \rho_{b} \widehat{\eta}\right) d \xi \\
& \sim \sum_{k \geq 0} \sum_{|\alpha|=k}\left[\int_{\mathbb{R}_{\xi}} e^{i \tau \xi} D^{\alpha} a(0, y, \xi, 0) d \xi\right] \widehat{\eta}^{\alpha} \rho_{b}^{k} \\
& \sim \sum_{|\alpha| \geq 0}\left[\int_{\mathbb{R}_{\xi}} e^{i \tau \xi} D^{\alpha} a(0, y, \xi, 0) d \xi\right] \eta^{\alpha}
\end{aligned}
$$

(with $\widehat{\eta} \in S^{*} \partial X$ and $\eta \in T^{*} \partial X$ ) hence has the form described in the theorem.

Conversely, any such formal power series can be asymptotically summed. Namely one can first get the symbol right, by taking Taylor series for the symbols, and then correcting the series for the smoothing terms. Thus the map is surjective. That is, given $\mathcal{K}_{\alpha} \in I_{\mathcal{S}}^{k^{\prime}-|\alpha|}\left(\mathcal{I}_{\partial X}\right)$, we can find $a(0, y, \xi, \eta)$ as in (1.16) such that

$$
\int_{\mathbb{R}_{\xi}} e^{i \tau \xi} a(0, y, \xi, \eta) d \xi \sim \sum_{|\alpha| \geq 0} \mathcal{K}_{\alpha} \eta^{\alpha} .
$$

This condition only restricts the behavior of $a$ near $(0, y, \xi, \eta)$, hence the vanishing of the full b-symbol does not restrict the possible cusp-symbols. Finally, an element of the null space has, by (1.13), $b, c$ symbol vanishing to infinite order, and so is given by an (arbitrary) family in $\rho_{b}^{\infty} \rho_{\mathrm{cu}}^{-k} \Psi_{\mathrm{b}, \mathrm{cu}}^{-\infty}\left(\mathcal{I} / S^{*} \partial X ; E, F\right)$.

\section{K-THEORY}

2.1. Smooth K-theory. We define K-theory groups of zero operators as smooth versions of the K-theory groups of the symbol algebra. Following [26. Definition 2], set

$$
A_{0}(M ; \mathbb{E})=\left\{(\sigma(A), \mathcal{N}(A)): A \in \Psi_{0}^{0}(M ; \mathbb{E}) \text { Fredholm on } L^{2}\right\}
$$

for a superbundle $\mathbb{E}=\left(E^{+}, E^{-}\right)$and then define $\mathcal{K}_{0}^{0}(M)$ to consist of the equivalence classes of elements of the union of the $A_{0}(M ; \mathbb{E})$ over $\mathbb{E}$, where two elements are equivalent if there is a finite chain consisting of one of the the following three equivalence notions. First,

$$
A_{0}(M ; \mathbb{E}) \ni(\sigma, N) \sim\left(\sigma^{\prime}, N^{\prime}\right) \in A_{0}(M ; \mathbb{F})
$$

if there are bundle isomorphisms $\mathbb{E}^{ \pm} \longrightarrow \mathbb{F}^{ \pm}$over $M$, that intertwine $(\sigma, N)$ and $\left(\sigma^{\prime}, N^{\prime}\right)$. Secondly,

$$
A_{0}(M ; \mathbb{E}) \ni(\sigma, N) \sim(\widetilde{\sigma}, \tilde{N}) \in A_{0}(M ; \mathbb{E})
$$


if there exists a homotopy of Fredholm operators $A_{t}$ in $\Psi_{0}^{0}(M ; \mathbb{E})$, with $(\sigma, N)=\left(\sigma\left(A_{0}\right), \mathcal{N}\left(A_{0}\right)\right)$ and $(\widetilde{\sigma}, \widetilde{N})=\left(\sigma\left(A_{1}\right), \mathcal{N}\left(A_{1}\right)\right)$, and finally

$$
A_{0}(M ; \mathbb{E}) \ni(\sigma, N) \sim\left(\sigma \oplus \operatorname{Id}_{F}, N \oplus \operatorname{Id}_{F}\right) \in A_{0}(M ; \mathbb{E} \oplus F)
$$

where $\mathbb{E} \oplus F=\left(E^{+} \oplus F, E^{-} \oplus F\right)$. Similarly, we define $\mathcal{K}_{0}^{1}(M)$ as consisting of the equivalence classes of elements in the suspended space (corresponding to based loops)

$$
A_{0, \text { sus }}(M ; E)=\left\{s \in C^{\infty}\left(\mathbb{R}, A_{0}(M ; E)\right): s(t)-\operatorname{Id}_{E} \in \mathcal{S}\left(\mathbb{R} ; \Psi_{0}^{0}(X ; E)\right\},\right.
$$

where $E=(E, E)$ as a superbundle and the equivalence condition is a finite chain of relations (2.1), (2.2), (2.3) with bundle transformations and homotopies required to be the identity to infinite order at $\pm \infty$.

Similarly, we define $\mathcal{K}_{-\infty, 0}^{0}(M)$ and $\mathcal{K}_{-\infty, 0}^{1}(M)$ as the groups of the corresponding equivalence classes of elements in

$$
A_{-\infty, 0}(M ; E)=\left\{\mathcal{N}(\mathrm{Id}+A): A \in \Psi_{0}^{-\infty}(M ; E), \mathrm{Id}+A \text { Fredholm on } L^{2}\right\},
$$

and

$A_{-\infty, 0, \text { sus }}(M ; E)=\left\{s \in C^{\infty}\left(\mathbb{R}, A_{-\infty, 0}(M ; E)\right): s-\mathrm{Id} \in \mathcal{S}\left(\mathbb{R} ; \Psi_{0}^{-\infty}(X ; E)\right\}\right.$

respectively.

2.2. K-theory sequence. From the compatibility of these two definitions and the standard definition of (absolute) K-theory with compact supports it follows directly that there is a sequence of maps

$$
\mathcal{K}_{-\infty, 0}^{i}(M / B) \longrightarrow \mathcal{K}_{0}^{i}(M / B) \longrightarrow \mathrm{K}_{\mathrm{c}}^{i}\left(T^{*}(M / B)\right)
$$

which is shown below to be exact.

2.3. $C^{*} \mathbf{K}$-theory. It is perhaps not immediately apparent that the definitions above lead to groups, but this follows from the approximation properties discussed below. In fact these also show that $\mathcal{K}_{0}^{i}(X)$ is closely related to the K-theory of the opposite parity of quotient of the $C^{*}$ closure of the algebra $\Psi_{0}^{0}(X)$ by the compact operators. Although we will not use this below we discuss this connection in order to relate our results to those in the literature.

We start by fixing some notation, define

$$
\begin{aligned}
& \mathcal{F}_{0}(X ; \mathbb{E})=\left\{A \in \Psi_{0}^{0}(X ; \mathbb{E}): A \text { is Fredholm on } L_{0}^{2}(X ; \mathbb{E})\right\}, \\
& \overline{\mathcal{F}_{0}(X ; \mathbb{E})}=\left\{A \in \bar{\Psi}_{0}^{0}(X ; \mathbb{E}): A \text { is Fredholm on } L_{0}^{2}(X ; \mathbb{E})\right\},
\end{aligned}
$$

where $\bar{\Psi}_{0}^{0}(X ; \mathbb{E})$ is the $C^{*}$-closure of $\Psi_{0}^{0}(X ; \mathbb{E})$ as operators on $L_{0}^{2}(X ; \mathbb{E})$. Thus $A_{0}(X ; \mathbb{E})$ is just the symbol data of elements of $\mathcal{F}_{0}(X ; \mathbb{E})$. Also denote 
by $\mathfrak{K}$ the compact operators acting on $L_{0}^{2}(X)$. The group $\mathcal{K}_{0}^{0}(X)$ defined above is closely related to the group

$$
\begin{aligned}
K_{1}^{C^{*}}\left(\bar{\Psi}_{0}^{0}(X) / \mathfrak{K}\right) & =\lim _{\rightarrow} \mathrm{GL}_{n}\left(\bar{\Psi}_{0}^{0}(X) / \mathfrak{K}\right) / \mathrm{GL}_{n}\left(\bar{\Psi}_{0}^{0}(X) / \mathfrak{K}\right)_{0} \\
& =\lim _{\rightarrow} \overline{\mathcal{F}_{0}\left(X ; \mathbb{C}^{n}\right)} / \sim
\end{aligned}
$$

where $\sim$ denotes homotopy. Indeed this will follow immediately from the following lemma of Atiyah.

Lemma 2.1 ([2], Lemma A9). Let $L \stackrel{\pi}{\rightarrow} M$ be a continuous linear map of Banach spaces with $\pi(L)$ dense in $M$ and let $U \subseteq M$ be open. Then for any compact $T$ the induced map

$$
\left[T, \pi^{-1}(U)\right] \rightarrow[T, U] \text { is bijective. }
$$

Remark. The proof of the lemma does not use that $L$ is complete, only that it is a normed vector space over a complete field.

Proposition 2.2. The group $K_{1}^{C^{*}}\left(\bar{\Psi}_{0}^{0}(X) / \mathfrak{K}\right)$ is isomorphic to $\lim _{\rightarrow} \mathcal{F}_{0}\left(X ; \mathbb{C}^{n}\right) / \sim$ and fits into the short exact sequence

$$
0 \rightarrow K_{1}^{C^{*}}(C(X)) \rightarrow K_{1}^{C^{*}}\left(\bar{\Psi}_{0}^{0}(X) / \mathfrak{K}\right) \rightarrow \mathcal{K}_{0}^{0}(X) \rightarrow 0 .
$$

Proof. It is clear that every equivalence class in $\overline{\mathcal{F}_{0}\left(X ; \mathbb{C}^{n}\right)} / \sim$ has a representative from $\mathcal{F}_{0}\left(X ; \mathbb{C}^{n}\right)$ and Atiyah's lemma shows that elements of $\mathcal{F}_{0}\left(X ; \mathbb{C}^{n}\right)$ are homotopic in $\mathcal{F}_{0}\left(X ; \mathbb{C}^{n}\right)$ if and only if they are homotopic in $\overline{\mathcal{F}_{0}\left(X ; \mathbb{C}^{n}\right)}$. Thus the groups $\mathcal{F}_{0}\left(X ; \mathbb{C}^{n}\right) / \sim$ and $\overline{\mathcal{F}_{0}\left(X ; \mathbb{C}^{n}\right)} / \sim$ are the same.

Exactness of the sequence follows from the fact that every non-compact manifold has a nowhere vanishing vector field (see e.g., [19, Proposition 9] for a proof). In particular, for a Fredholm operator in $\Psi_{0}^{0}(X ; \mathbb{E})$ we always have $E^{+} \cong E^{-}$. So by simultaneously stabilizing $E^{+}$and $E^{-}$we see that every class in $\mathcal{K}_{0}^{0}(X)$ has a representative in $\mathcal{F}_{0}\left(X ; \mathbb{C}^{n}\right)$ and two representatives differ by a bundle isomorphism. Finally, the existence of a non-vanishing section also implies that the map $K_{1}^{C^{*}}(C(X)) \rightarrow K_{1}^{C^{*}}(\bar{\Psi} / \mathfrak{K})$ is injective. Indeed, if $\Phi$ represents a class in $K_{1}^{C^{*}}(C(X))$ and $\Phi$ is homotopic to the identity through Fredholm operators in $\Psi_{0}^{0}\left(X ; \mathbb{C}^{n}\right)$ then the principal symbol of any such homotopy evaluated at a non-vanishing section shows that $\Phi$ represents the identity in $K_{1}^{C^{*}}(C(X))$.

A similar statement is true for the odd smooth K-theory group, $\mathcal{K}_{0}^{1}(X)$. Our computations hold more generally for families of operators acting on a fibration $M \rightarrow B$ for which the analogue of the $C^{*} \mathrm{~K}$-theory groups are the $K(B)$-linear $K K$ groups mentioned in the introduction. 


\section{Relation Between B AND CUSp CAlCuli}

In this section, we briefly review from [9], 23] the relations between the $\mathrm{b}$ and cusp calculi. We establish that the K-theory of the b-calculus is universal as described in the introduction. For later use, we show that the group of invertible elements in the $b, c$ calculus of order $-\infty$ vanishing to infinite order at the cusp end form a contractible semigroup.

Recall that, on any manifold with boundary, $X$, the Lie algebra of bvector fields, $\mathcal{V}_{\mathrm{b}}$, consists of those vector fields tangent to the boundary and in terms of local coordinates $x, z_{i}$ ( $x$ a boundary defining function) near the boundary is spanned by $x \partial_{x}, \partial_{z_{i}}$. Similarly, for an admissible choice of $x$ for the cusp structure, the Lie algebra of cusp vector fields, $\mathcal{V}_{\text {cu }}(X)$ is locally spanned by $x^{2} \partial_{x}, \partial_{z_{i}}$.

The (canonical) b-structure on a manifold with boundary $X$ induces a

cusp-structure on $\widetilde{X}$, a manifold diffeomorphic to $X$ but with the smooth structure enlarged to include the new boundary defining function

$$
r=\mathrm{ilg}(x)=\frac{1}{\log \frac{1}{x}} .
$$

Thus a function $f$ is smooth on $\tilde{X}$ if it is smooth in the interior and near the boundary is of the form

$$
f\left(r, z_{i}\right)=g\left(\operatorname{ilg}(x), z_{i}\right)
$$

for some function $g \in C^{\infty}([0,1) \times \partial X)$. The identity map $\tilde{X} \longrightarrow X$ is then smooth, corresponding to the natural inclusion

$$
C^{\infty}(X) \longrightarrow C^{\infty}(\widetilde{X})
$$

but in the opposite direction the identity map is not smooth. In this sense, $\widetilde{X}$ has 'more' smooth functions than $X$ (although it is diffeomorphic to it, but not naturally so). Both the interiors and the boundaries of $X$ and $\widetilde{X}$ are canonically identified - the manifolds only differ in the manner in which the boundary is attached (cf. [9], [8, §2]).

Now $\mathfrak{i}_{\text {ilg }}^{*}\left(x \partial_{x}\right)=r^{2} \partial_{r}$ and indeed, $\mathfrak{i}_{\text {ilg }}^{*} \mathcal{V}_{\mathrm{b}}(X)$ spans $\mathcal{V}_{\mathrm{cu}}(\widetilde{X})$ over $\mathcal{C}^{\infty}(\widetilde{X})$. The induced relation between $b$ and cusp differential operators extends to a close relationship between the corresponding pseudodifferential operators. In fact, the functor $X \rightarrow X_{\mathrm{lt}}$ (consisting of blowing-up all of the boundary faces logarithmically and then 'classically') takes the b-stretched double space of $X$ to the cusp-stretched double space of $\tilde{X}$, preserving composition [9, §2.5-2.6, (4.4), §4.6]. This leads to the following result, the first part of which is [22, Prop. 26]. The second part is just the observation that the lower order parts of the expansion of the kernel of an operator at the front face can be smoothly deformed away without changing the normal operator and preserving ellipticity.

\section{Proposition 3.1.}


i) The lift $\left(\beta_{l t}^{2}\right)^{*} \Psi_{b}^{s}(X)$ is a subcalculus of $\Psi_{\mathrm{cu}}^{s}(\widetilde{X})$ with dense span over $C^{\infty}(\widetilde{X})$.

ii) Any fully elliptic element of $\Psi_{\mathrm{cu}}^{s}(\widetilde{X})$ is homotopic through fully elliptic elements to an element in $\left(\beta_{l t}^{2}\right)^{*} \Psi_{b}^{s}(X)$.

Corollary 3.2. The K-theory groups of the $b$ and cusp calculi are naturally isomorphic,

$$
\mathcal{K}_{-\infty, \mathrm{b}}^{*}(M / B) \cong \mathcal{K}_{-\infty, \mathrm{cu}}^{*}(M / B), \mathcal{K}_{\mathrm{b}}^{*}(M / B) \cong \mathcal{K}_{\mathrm{cu}}^{*}(M / B) ;
$$

since the cusp calculus is 'universal' [26], so is the b-calculus.

As in 25$]$ we set

$$
\begin{aligned}
& \dot{G}^{-\infty}(X ; E)=\left\{\text { elements of } \operatorname{Id}+\dot{\Psi}^{-\infty}(X ; E) \text { invertible on } L^{2}\right\}, \\
& G_{\mathcal{S}}^{-\infty}(X ; E)=\left\{\text { elements of } \operatorname{Id}+\Psi_{\mathcal{S}}^{-\infty}(X ; E) \text { invertible on } L^{2}\right\},
\end{aligned}
$$

and then the images of the normal operators on $G_{\mathrm{cu}}^{-\infty}(X ; E)$ and $G_{\mathrm{b}}^{-\infty}(X ; E)$ are the corresponding subgroups on which the index vanishes; they will be denoted

$$
G_{\text {sus,ind }=0}^{-\infty}(\partial X ; E), G_{I, \text { ind }=0}^{-\infty}(\partial X ; E)
$$

respectively. An element of the former can be smoothly deformed to an element of the latter thanks to Proposition 3.1(ii).

In particular for an interval, we will also need the case of a manifold with disconnected boundary, say

$$
\partial X=\partial X_{0} \sqcup \partial X_{1}
$$

and the subgroup $G_{\mathrm{b}}^{-\infty, \infty}(X ; E)$ in $G_{\mathrm{b}}^{-\infty}(X ; E)$ consisting of those operators which are perturbations of the identity with kernels vanishing to infinite order at the corner $\partial X_{1} \times \partial X_{1}$.

Corollary 3.3. The semigroups $G_{b}^{-\infty}(X ; E)$ and $G_{b}^{-\infty, \infty}(X ; E)$ are weakly contractible.

Proof. The proof of [25, Lemma 1], that the sequence

$$
\dot{G}^{-\infty}(X ; E) \longrightarrow G_{\mathrm{cu}}^{-\infty}(X ; E) \stackrel{I_{\mathrm{cu}}}{\longrightarrow} G_{\text {sus }, \text { ind }=0}^{-\infty}(\partial X ; E)
$$

is a Serre fibration, produces a lift, $h_{t}^{\prime}$, of any family

$$
h_{t}: \mathcal{I}^{k} \longrightarrow G_{\text {sus,ind }=0}^{-\infty}(\partial X ; E)[[x]], t \in[0,1]
$$

to $G_{\mathrm{cu}}^{-\infty}(X ; E)$ with the convenient property that $I_{\mathrm{cu}}\left(h_{t}^{\prime}\right)=h_{t}$. This construction does not make use of the group structure of $G_{\mathrm{cu}}^{-\infty}(X ; E)$ and so holds verbatim for the sequence

$$
\dot{G}^{-\infty}(X ; E) \longrightarrow G_{\mathrm{b}}^{-\infty}(X ; E) \stackrel{I_{\mathrm{b}}}{\longrightarrow} G_{I, \text { ind }=0}^{-\infty}(\partial X ; E)
$$

and for the restriction

$$
\dot{G}^{-\infty}(X ; E) \longrightarrow G_{\mathrm{b}}^{-\infty,-\infty}(X ; E) \stackrel{I_{\mathrm{b}}}{\longrightarrow} G_{I, \text { ind }=0}^{-\infty}\left(\partial X_{0} ; E\right)
$$


when $\partial X=\partial X_{0} \sqcup \partial X_{1}$.

Passage to $\widetilde{X}$ relates the two sequences by the commutative diagram

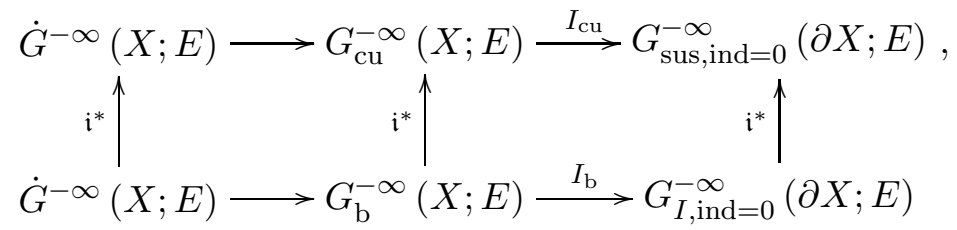

and both the top and bottom rows induce long exact sequences of homotopy groups. Since the left and right vertical arrows above are homotopy equivalences, they induce isomorphisms of the corresponding homotopy groups. The Five Lemma then shows that the middle vertical arrow is a weak homotopy equivalence between $G_{\mathrm{b}}^{-\infty}(X ; E)$ and the contractible space $G_{\mathrm{cu}}^{-\infty}(X ; E)$. A similar argument shows the weak contractibility of $G_{\mathrm{b}}^{-\infty,-\infty}(X ; E)$.

We remark that since $G_{\mathrm{b}}^{-\infty}(X ; E)$ is dominated by a CW-space, the Whitehead lemma implies that $\mathfrak{i}^{*}$ is actually a homotopy equivalence and hence that $G_{\mathrm{b}}^{-\infty}(X ; E)$ is contractible [27, Prop. 8.3]; we do not use this in the sequel.

\section{Fredholm perturbations of the identity}

In this section we identify the groups $\mathcal{K}_{-\infty, 0}^{*}(M / B)$ with the topological K-theory groups of the cotangent bundle of the boundary.

We quickly review the relevant definitions in terms of the groups defined in (3.1). Recall that for any manifold, $X, K^{1}(X)$ can be realized as stable homotopy classes of maps into GL( $N)$ or more directly (see [25, Prop. 3]) in terms of a classifying space

$$
\mathrm{K}^{1}(X)=\lim _{\rightarrow}[X ; \mathrm{GL}(N)]=\left[X ; \dot{G}^{-\infty}(V ; E)\right],
$$

with $V$ any manifold with boundary. Correspondingly (see [25, Prop. 4])

$$
\mathrm{K}^{0}(X)=\lim _{\rightarrow}\left[X ; C^{\infty}\left(\left(\mathbb{S}^{1}, 1\right) ;(\mathrm{GL}(N), \mathrm{Id})\right)\right]=\left[X ; G_{\mathrm{sus}}^{-\infty}(U ; E)\right],
$$

with $U$ any closed manifold of positive dimension. For a compact manifold with boundary these definitions give the absolute K-theory which we denote (somewhat unconventionally) in the same way. The compactly supported K-theory $\mathrm{K}_{\mathrm{c}}^{*}(Y)$ of a non-compact manifold (possibly with boundary) is defined in the same way, except that maps and homotopies are required to reduce to the identity outside some compact subset of $Y$.

Theorem 4.1. For any fibration with fibres which are compact manifolds with boundary there is a canonical isomorphism

$$
\mathcal{K}_{-\infty, 0}^{*}(M / B) \longrightarrow \mathrm{K}_{c}^{*}\left(T^{*} \partial(M / B)\right) .
$$


Proof. For simplicity of notation we first consider the case that there is only a single compact manifold with boundary, $X$. Given an element $\kappa \in$ $\mathcal{K}_{-\infty, 0}^{0}(X)$, represented by $\mathrm{Id}+A$ with $A \in \Psi_{0}^{-\infty}(X ; E)$ we proceed to associate to it an element $r(\kappa) \in \mathrm{K}_{\mathrm{c}}^{0}\left(T^{*} \partial X\right)$. First, by adding the identity on a complementary bundle, we may stabilize $E$ to $\mathbb{C}^{M}$ for some large enough $M$ without changing $\kappa$. In fact $\kappa$ is determined by the normal operator of $\mathrm{Id}+A$, and hence its reduced normal operator, $\mathrm{Id}+\alpha$.

The condition that $\mathrm{Id}+A$ be Fredholm is equivalent to the invertibility (on $L^{2}$ ) of $\mathrm{Id}+\alpha$ as a family of operators on an interval bundle over $S^{*} X$. Proposition 1.1 characterizes the range of the reduced normal operator in the calculus of b-pseudodifferential operators of order $-\infty$ on the interval (and trivial at one end). Let us denote by

$$
\mathcal{H}^{-\infty}(X ; E) \subset \rho_{\mathrm{cu}}^{\infty} \Psi_{\mathrm{b}, \mathrm{cu}}^{-\infty}\left(\mathcal{I} / S^{*} \partial X ; E\right)
$$

the semigroup of those elements which are invertible. Corollary 3.3 shows that the fibres of $\mathcal{H}^{-\infty}(X ; E)$ are weakly contractible after stabilization. Thus, any section of $\mathcal{H}^{-\infty}(X ; E)$ is homotopic to the identity section after stabilization into $\mathcal{H}^{-\infty}\left(X ; \mathbb{C}^{M}\right)$ for sufficiently large $M$. In particular this is the case for $\alpha$, so there exists a smooth curve

$$
\begin{aligned}
\alpha^{\prime}:[0,1] \longrightarrow & \mathcal{H}^{-\infty}\left(X ; \mathbb{C}^{M}\right) \\
& \text { such that } \alpha^{\prime}(t)=\alpha \text { for } t<\epsilon, \alpha^{\prime}(t)=\operatorname{Id} \text { for } t>1-\epsilon
\end{aligned}
$$

for some $\epsilon>0$. This is a family of b-pseudodifferential operators on a bundle of intervals over $[0,1] \times S^{*}(\partial X)$ and of necessity the family of b-indicial families $\gamma(t)=I_{\mathrm{b}}\left(\alpha^{\prime}(t)\right)$ is also invertible as a family of matrices. Thus associated with $\alpha^{\prime}$ is a map

$$
\gamma \in \mathcal{C}^{\infty}\left(T^{*} \partial X, G_{\text {sus }}^{-\infty}\left(\{0\}, \mathbb{C}^{M}\right)\right) .
$$

Here we have used the fact from (1.10) that near $t=0$ the b-indicial family is independent of the fibre variables of $S^{*}(\partial X)$ and near $t=1$ it is, by construction, equal to the identity. Thus, the variable $t$ can be interpreted as a compactified radial variable for $T^{*}(\partial X)$ giving (4.6).

This defines the desired map

$$
\mathcal{K}_{-\infty, 0}^{0}(X) \ni \kappa \longmapsto[\gamma] \in \mathrm{K}_{\mathrm{c}}^{0}\left(T^{*} \partial X\right)
$$

which is to say that the K-class defined by $\gamma$ depends only on $\kappa$ and not the intermediate choices. As in [27, Prop 5.19], the final class is independent of the trivialization of $E$ and, since any two homotopies between sections of $\mathcal{H}^{-\infty}\left(X ; \mathbb{C}^{M}\right)$ are themselves homotopic after stabilization, it is independent of the choice of $\alpha^{\prime}$. Also, note that the product of two such elements $\operatorname{Id}+A$, $\mathrm{Id}+B$ is mapped to the product in $\mathrm{K}_{\mathrm{c}}^{0}\left(T^{*} \partial M\right)$ since we can take the product of the homotopies.

Now, it remains to show that (4.7) is an isomorphism. Surjectivity follows from the fact that every element in $\mathrm{K}_{\mathrm{c}}^{0}\left(T^{*} \partial X\right)$ may be represented by a family $\gamma(t)$ which is of the form discussed above, so is an invertible family 
of indicial operators, independent of the fibre variables near $t=0$. From Proposition 1.1 this may be quantized to an elliptic b,c operator of the form Id $+\alpha^{\prime}(t)$ (with $\alpha^{\prime}(t)$ trivial at the cusp end) reducing to the identity in $t>1-\epsilon$. The index bundle of such a family (see the discussion below) is trivial, hence it may be perturbed by a smoothing family (depending on all variables) to be invertible and this may be chosen to be in the range of the reduced normal operator for $t<\epsilon$. The result at $t=0$ corresponds to a fully elliptic operator $\mathrm{Id}+A$ in the zero calculus and hence a class $\kappa$ mapping to the given $\gamma$.

To see injectivity, consider a class $\kappa$ such that the construction above leads to a family (so for an appropriate choice of initial representative) which is homotopic to the identity, as a map on $T^{*} \partial X$. Then after a small perturbation it can be arranged that this homotopy is a family of the form of $\gamma$, thus there is a 2-parameter family of indicial operators $\gamma(t, s)$ on $[0,1]^{2}$ with $\gamma(t, 0)$ the indicial family and $\gamma(t, s)$ independent of the fibre variables for $s<\epsilon$ and reducing to the identity if either $s>1-\epsilon$ or $t>1-\epsilon$. Again from Proposition 1.1 there is no obstruction to 'quantizing' this to a family of reduced normal operators, for $s<\epsilon$ and to a family of b,c operators with more general dependence on $S^{*} \partial X$ in $s>\epsilon$. Such a family $\alpha^{\prime}(t, s)$ may be chosen to be the identity where $\gamma$ is the identity and to reduce to the original homotopy at $s=0$. Now, as an elliptic family, it has a virtual index bundle over $[0,1]^{2} \times S^{*} \partial X$. As a bundle this may be realized as the difference of the null and conull bundles for $\alpha^{\prime}(t, s)(\mathrm{Id}-\Pi)$ where $\Pi$ is a finite rank selfadjoint smoothing operator with range in $\dot{\mathcal{C}}^{\infty}(\mathcal{I})$ and sufficiently large rank. It follows that the index bundle is trivial and that the family may be made invertible by a similar perturbation. At $t=0$ this gives a homotopy within the range of the reduced normal operator to the identity. Thus the class $\kappa$ is trivial.

Although this proof is written out only in the case of a single manifold it is the dependence on the fibre variables in $S^{*} \partial X$ which is crucial and it carries over with only notational changes to the general setting of a fibration (11). The argument for the odd K-groups involves only the addition of a parameter.

\section{Six TERM EXACT SEQUENCE}

We start out with a theorem very similar to [26, Lemma 2.2] and to the Atiyah-Bott analysis of the index problem for boundary value problems [3]. We will exploit the similarity to [26] to identify a six-term exact sequence for the zero calculus with that of the scattering calculus.

Theorem 5.1. For any fibration (11) (with fibres modeled on a compact manifold with boundary) the smooth $K$-theory $\mathcal{K}_{0}^{i}(M / B)$ is represented by the Fredholm zero operators which are equal to the identity near the boundary 
and hence there is a natural isomorphism

$$
\mathcal{K}_{0}^{0}(M / B) \longrightarrow \mathrm{K}_{c}^{*}\left(T^{*}(\stackrel{\circ}{M} / B)\right) .
$$

Proof. Consider an element $A \in \Psi_{0}^{0}(M / B ; \mathbb{E})$ which is fully elliptic and hence represents an element of $\mathcal{K}_{0}^{0}(M / B)$. The reduced normal family of $A$ is an invertible (on $L^{2}$ ) element of the b,c calculus on an interval bundle over $S^{*}(\partial M / B)$. The symbol of $A, \sigma(A)$, is a bundle isomorphism between $E^{+}$and $E^{-}$over the zero cosphere bundle of the fibers of $M / B$. At the boundary, the inward normal fixes a section of this bundle so $\sigma(A)$ at this section may be used to identify $E^{+}$and $E^{-}$at, and hence near, the boundary. Thus, $\sigma(A)$ may be taken to be the identity at this section.

The indicial family at the b-front face, being invertible, then gives a section of the isomorphism bundle of the lift of $E$ to the whole of the compactified normal bundle to the boundary. By construction this section is the identity at the inward-pointing end and is consistent with the symbol at the outward-pointing end. So we may deform (within the b,c-calculus, not a priori within the range of the reduced normal operator) the b-indicial operator keeping the consistency with an elliptic symbol at the inward-pointing end until the b-indicial operator is the identity and the symbol is the identity near both inward- and outward-pointing normal sections. Now, from Proposition 1.2 this homotopy may realized in the image of the reduced normal operators of the zero calculus, so there is an elliptic curve $A_{t} \in \Psi_{0}^{0}(M / B ; \mathbb{E})$ with $A_{0}=A$ and such that $A_{1}$ has full b-indicial operator equal to the identity. Initially this curve need not be fully elliptic, but the reduced normal family is fully elliptic. Again by standard index theory this can be modified to an invertible family, without change near $t=0$, by adding a term of order $-\infty$ with support in the interior of the interval.

So, after this initial homotopy we conclude that $\mathcal{K}_{0}^{0}(M / B)$ is represented by fully elliptic families $A \in \Psi_{0}^{0}(M / B, \mathbb{E})$ where $E^{+}$and $E^{-}$are identified near the boundary and with symbol which is the identity near both normal directions and with reduced normal family having full b-indicial family equal to the identity. Thus the reduced normal family is in fact a section over $S^{*}(\partial(M / B))$ of the bundle of groups with fibre the group

$$
G_{\mathrm{cu}}^{\cdot,-\infty}(\mathcal{I} ; E)
$$

that is, trivial near the b-end. As explained in $\$ 3$, after stabilization this group is (weakly) contractible. Thus in fact the reduced normal section may be (after stabilization) contracted to the identity section. As above, this family may be lifted to a fully elliptic homotopy of $A$ to a family which is the identity near the boundary and in particular has reduced normal family equal to the identity.

Now the isomorphism (5.1) is just the usual Atiyah-Singer isomorphism for the symbol.

Corollary 5.2. With the smooth K-theory of the zero calculus identified with the compactly supported K-theory of the fibre cotangent bundle of the 
interior of $M$, the analytic index factors through the Atiyah-Singer index map for the double $2 M=M \cup M^{-}$

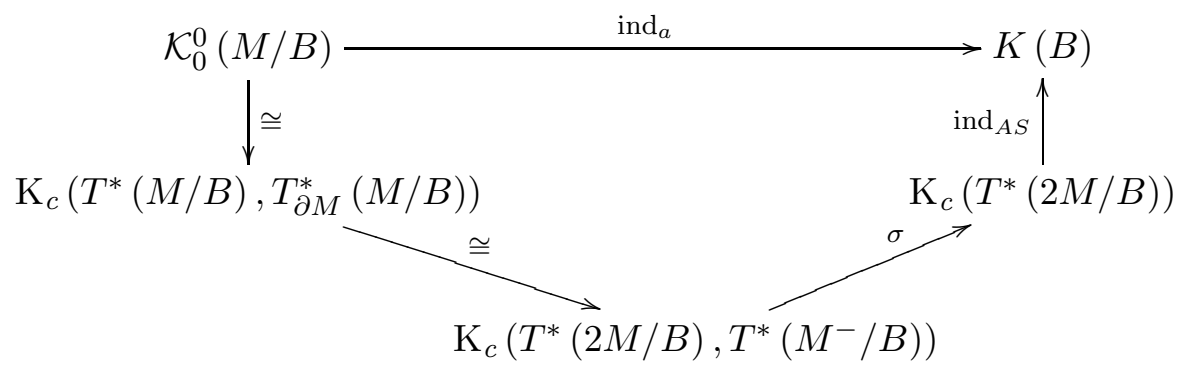

Proposition 5.3. The smooth K-theory groups of the zero calculus lead to the inner six term exact sequence

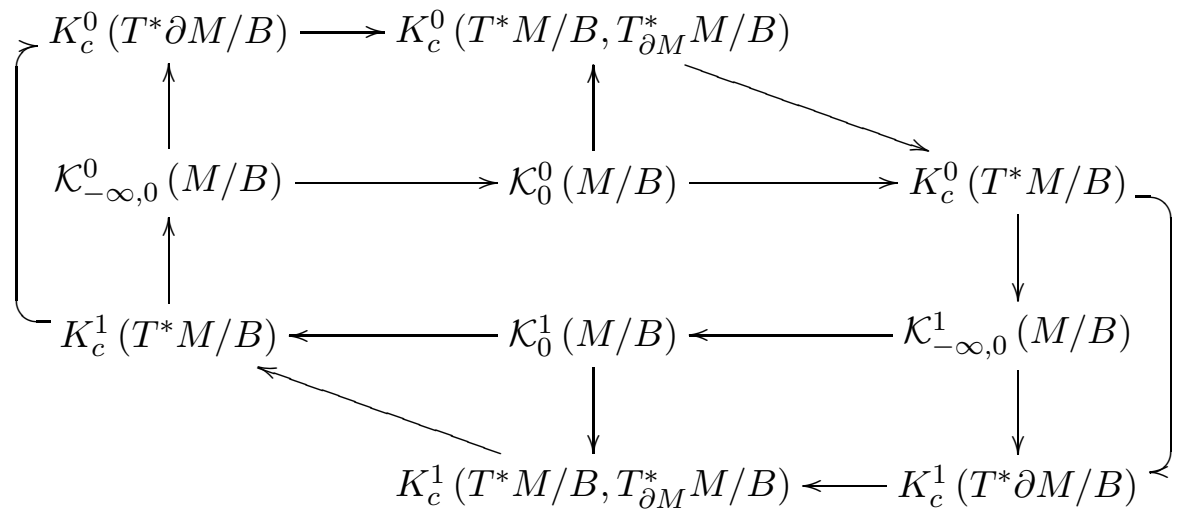

which is naturally isomorphic to the outer, geometric, sequence and thus the zero calculus is 'geometric', in the sense of the introduction.

Proof. The exactness of the inner diagram follows from the known exactness of the outer diagram and the commutativity of the whole, so it only remains to show the latter.

We can fit the maps from Theorems 4.1 and 5.1 into the diagram

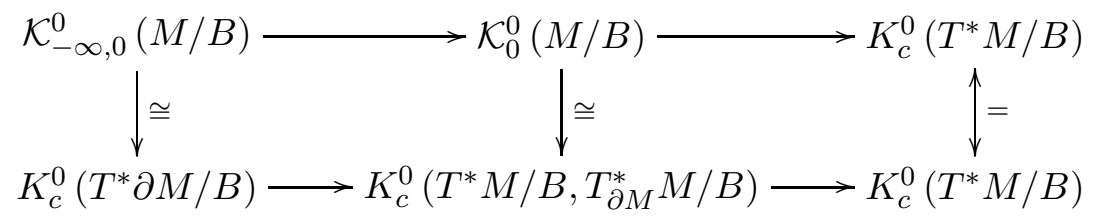

in which the right square clearly commutes. For the commutativity of the left hand square, note that the first map along the bottom is given by

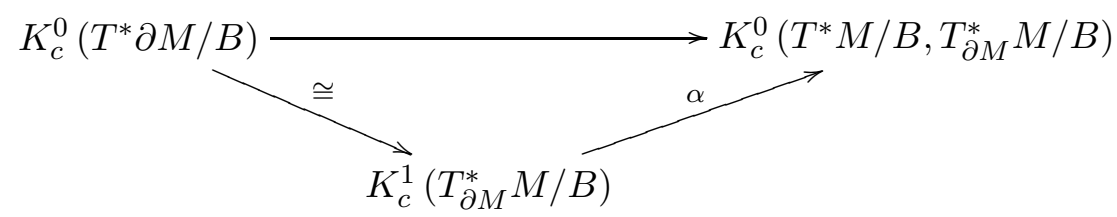


where $\alpha$ is defined by interpreting $T_{\partial M}^{*} M$ as a collar neighborhood of $T^{*} \partial M$ in $T^{*} M$, and the same collar neighborhood idea is used in the identification of $\mathcal{K}_{0}^{0}(M / B)$ and $K_{c}^{0}\left(T^{*} M / B, T_{\partial M}^{*} M / B\right)$. Indeed, given a representative $A$ of a class in $\mathcal{K}_{0}^{0}(M / B)$, we found a homotopy of the normal operators starting at $\mathcal{N}(A)$ and ending at the identity. We can think of this homotopy as happening along a collar neighborhood by identifying it with $T^{*} \partial X \times[0,1]_{\varepsilon}$ and using $\varepsilon$ as the homotopy variable. Finally, note that the identification of $\mathcal{K}_{-\infty, 0}^{0}(M / B)$ with $K_{c}^{0}\left(T^{*} \partial M / B\right)$ was also by means of a homotopy (this time thinking of $T^{*} \partial M / B$ as $S^{*} \partial M / B \times \mathbb{R}^{+}$and running the homotopy in $\mathbb{R}^{+}$) and so we can see that the left square also commutes.

The same arguments show the commutativity of the corresponding squares involving the odd K-theory groups. The two remaining squares, involving $\mathrm{K}_{\mathrm{c}}{ }^{*}\left(T^{*} M / B\right) \rightarrow \mathcal{K}_{-\infty, 0}^{*+1}(M / B)$, commute by the definition of this map.

We point out that this proposition answers one of the questions from the introduction. Namely, given an elliptic symbol when is there a Fredholm family of operators in the zero calculus with that symbol? Since this is the same as asking if the symbol is in the image of the map

$$
\mathcal{K}_{0}^{0}(M / B) \rightarrow K_{c}^{0}\left(T^{*} M / B\right)
$$

in the diagram above, exactness of the diagram at $K_{c}^{0}\left(T^{*} M / B\right)$ answers this question completely.

Corollary 5.4. A family of operators in $\Psi_{0}^{0}(M / B ; \mathbb{E})$ can be perturbed by a family of operators in $\Psi_{0}^{-\infty}(M / B ; \mathbb{E})$ to be Fredholm if and only if its interior symbol is in

$$
\operatorname{ker}\left(K^{0}\left(T^{*} M / B\right) \rightarrow K^{1}\left(T_{\partial M}^{*} M / B\right)\right),
$$

i.e. , if its Atiyah-Bott obstruction vanishes. In which case, after stabilization, there is a perturbation that makes it invertible.

\section{RELATion to Boundary VAlue PROBlems}

Finally we relate the various approaches to quantization mentioned in the introduction: the scattering calculus, the zero calculus, elliptic boundary value problems as discussed by Atiyah and Bott, and the transmission algebra of Boutet de Monvel. We will confine ourselves to relating the approaches to the index problem, as a complete study of the relations between these various calculi is more involved.

6.1. Atiyah-Patodi-Singer versus local boundary conditions. We start by recalling the general context of elliptic boundary value problems, initially for a single differential operator of first order. See for instance the exposition for the case of a single Dirac operator in [5].

Given an elliptic first order operator $P: \mathcal{C}^{\infty}(X ; E) \rightarrow \mathcal{C}^{\infty}(X ; F)$ on a compact manifold $X$ with non-trivial boundary, its kernel is infinite dimensional and obtaining a Fredholm operator requires a restriction reducing 
this to a finite dimensional space. The Calderón projector of $P, \Pi_{P}$, is the orthogonal projection onto the space of Cauchy data of $P$,

$$
C D(P)=\left\{s \iota_{\partial X}: s \in \mathcal{C}^{\infty}(X ; E), P s=0\right\} .
$$

An elliptic boundary condition is a pseudodifferential operator of order zero taking values in some auxiliary bundle $G$,

$$
\mathcal{C}^{\infty}\left(\partial X ; E \operatorname{lox}_{\partial} \stackrel{R}{\rightarrow} \mathcal{C}^{\infty}(\partial X ; G),\right.
$$

satisfying two conditions:

i) $H^{(s)}\left(\partial X ; E \iota_{\partial X}\right) \stackrel{R^{(s)}}{\longrightarrow} H^{(s)}(\partial X ; G)$ has closed range for every $s$

ii) $\operatorname{Ran}(\sigma(R))=\operatorname{Ran}\left(\sigma(R) \sigma\left(\Pi_{P}\right)\right) \cong \operatorname{Ran}\left(\sigma\left(\Pi_{P}\right)\right)$.

Given an elliptic boundary condition the corresponding 'realization' of $P$, $P_{R}$, given by restricting $P$ to

$$
\operatorname{Dom}\left(P_{R}\right)=\left\{s \in \operatorname{Dom}(P): R\left(\left.s\right|_{\partial X}\right)=0\right\}
$$

is a Fredholm operator [5, Thm 20.8, Prop. 18.16].

Two types of elliptic boundary conditions stand out. An elliptic condition is of generalized Atiyah-Patodi-Singer or APS type if $R$ is an orthogonal projection with the same principal symbol as $\Pi_{P}$. APS type boundary conditions always exist, e.g., $R=\Pi_{P}$.

An elliptic boundary condition is local if $\sigma(R)$ is surjective. In this case, condition $(i)$ above is automatic [5, Lemma 20.9]. The index of a local elliptic boundary problem only depends on the principal symbols of $P$ and $R$. This is not true for general, e.g. APS type, elliptic boundary conditions.

In 24 and 14, 13, the relation between elliptic boundary conditions of APS type and Fredholm b-pseudodifferential operators was analysed, leading to

Theorem 6.1 ([24], Lemma 5; [13], Theorem 1.1). Let $\mathrm{\partial}_{T}$ be a generalized Dirac-type operator on a manifold with boundary with boundary conditions of APS type, then there is a b-operator of order $-\infty, \mathcal{T}$, such that $\partial+\mathcal{T} \in$ $\Psi_{b}^{1}(M ; E)$ is a Fredholm operator with the same index as $\widetilde{\partial}_{T}$.

6.2. Atiyah-Bott obstruction. For a differential operator, $P$, of general degree, $k$, the Cauchy data involves the $k-1$ normal jet bundle at the boundary. It is standard (e.g., [10, Chapter X], [4, §II.6]) to study this space by passing to a family of ordinary differential operators formed from the principal symbol. This family, $P_{y, \widehat{\eta}}$, acts on half-lines and is parameterized by the cosphere bundle of the boundary. The ellipticity of $P$ gives rise to a decomposition of the null space of the $P_{y, \widehat{\eta}}$

$$
\mathcal{M}_{(y, \widehat{\eta})}=\mathcal{M}_{(y, \widehat{\eta})}^{+} \oplus \mathcal{M}_{(y, \widehat{\eta})}^{-}
$$

where $\mathcal{M}_{(y, \eta)}^{ \pm}$consists only of exponential polynomials involving $e^{i \lambda x}$ with $\pm \operatorname{Im}(\lambda)>0$ respectively. A local boundary condition defines a bundle map

$$
\beta^{+}: \mathcal{M}^{+} \rightarrow \pi^{*}(G) \text {. }
$$


and the Lopatinskii-Schapiro condition for ellipticity is that this map be an isomorphism.

Atiyah and Bott [1], 3] showed that the existence of elliptic boundary conditions, in the sense of Lopatinskii-Schapiro, for a given differential operator, $P$, is topologically obstructed in that the symbol of $P$ is then in the kernel of the map

$$
\mathrm{K}_{\mathrm{c}}\left(T^{*} X\right) \stackrel{r_{*}}{\rightarrow} \mathrm{K}_{\mathrm{c}}\left(T_{\partial X}^{*} X\right) .
$$

The isomorphism $\beta^{+}$then determines a lifting of $[\sigma(P)] \in \mathrm{K}_{\mathrm{c}}\left(T^{*} X\right)$ to $[\sigma(P), \sigma(R)] \in K\left(T^{*} X, T_{\partial X}^{*} X\right)$.

6.3. Boutet de Monvel transmission algebra. Boutet de Monvel [6] constructed an algebra of pseudodifferential which includes the generalized inverses of Lopatinskii-Schapiro elliptic boundary problems. A general element of the algebra acts between bundles $E_{1}, E_{2}$ over $X$ and bundles $F_{1}$, $F_{2}$ over $\partial X$ and is given by

$$
\mathcal{A}=\left(\begin{array}{cc}
P_{+}+G & K \\
T & Q
\end{array}\right): \begin{gathered}
\mathcal{C}^{\infty}\left(X ; E_{1}\right) \\
\mathcal{C}^{\infty}\left(\partial X ; F_{1}\right)
\end{gathered} \longrightarrow \begin{gathered}
\mathcal{C}^{\infty}\left(X ; E_{2}\right) \\
\mathcal{C}^{\infty}\left(\partial X ; F_{2}\right)
\end{gathered}
$$

where $P$ is a pseudodifferential operator satisfying the transmission condition, $P_{+}$is its action on $\mathcal{C}^{\infty}\left(X ; E_{1}\right)$ by extension-as-zero and restriction, $G$ is a 'singular Green operator', $K$ and $T$ are potential and trace operators, and $Q$ is a pseudodifferential operator on the boundary. A differential operator $P$ with local boundary condition can be realized as the operator

$$
\left(\begin{array}{l}
P \\
R
\end{array}\right)
$$

in the Boutet de Monvel algebra.

We will say that $\mathcal{A}$ is elliptic if $P$ is elliptic and fully elliptic if $\mathcal{A}$ defines a Fredholm operator on the natural Sobolev spaces. The Lopatinski-Schapiro conditions on $R$ are equivalent to asking that (6.2) be fully elliptic. With each operator $\mathcal{A}$ as in (6.1) is associated a 'boundary symbol' which is a family of operators of Wiener-Hopf type. Ellipticity of $P$ implies that this family is Fredholm and its index bundle is isomorphic to $\mathcal{M}^{+}$[6, pg. 35]. The operator $\mathcal{A}$ is fully elliptic if and only if it is elliptic and its boundary symbol is invertible.

By [6, Theorem 5.14], an elliptic pseudodifferential operator $P$ satisfying the transmission condition has a realization as a Fredholm operator $\mathcal{A}$ if and only if the Atiyah-Bott obstruction vanishes. Boutet de Monvel showed that any such fully elliptic operator is homotopic, through fully elliptic operators, to an element of the form

$$
\left(\begin{array}{cc}
P_{+}^{\prime} & 0 \\
0 & Q^{\prime}
\end{array}\right)
$$

where, moreover, $P_{+}^{\prime}$ is equal to the identity in a neighborhood of the identity. 
Melo, Schick, and Schrohe [20], following work of Melo, Nest, and Schrohe [19] showed that the K-theory of the $C^{*}$-closure of the symbol algebra in the Boutet de Monvel calculus, $\mathcal{K}_{*}$, gives a short exact sequence

$$
0 \rightarrow K_{*}(C(X)) \rightarrow \mathcal{K}_{*} \rightarrow K_{*+1}\left(T^{*} X^{o}\right) \rightarrow 0
$$

and the analytic index map factors through $K_{*+1}\left(T^{*} X^{o}\right)$.

Boutet de Monvel [6, pg. 33] explicitly mentions an extension to families of operators, proving that there exists an elliptic Green system $\mathcal{A}_{b}$ (depending continuously on $b \in B$ ) associated to $P_{b}$ if and only if $j\left(P_{b}^{+}\right) \in$ $K\left(S^{*} \partial X \times B\right)$ is the pull-back of a virtual bundle on $\partial X \times B$.

6.4. Relationship to scattering and zero calculi. The similarities between the three calculi mentioned in the introduction is striking given the disparate motivations behind them. Whereas the Boutet de Monvel transmission algebra was constructed precisely to deal with boundary value problems, the zero and scattering calculus serve to model certain asymptotically regular, non-compact manifolds which admit a smooth compactification to manifolds with boundary. The zero calculus is used to model asymptotically hyperbolic geometries and the scattering, asymptotically Euclidean. Nevertheless, the relation between the fully elliptic elements of the calculi is very close.

Theorem 6.2. For an element $p \in \mathrm{K}_{c}\left(T^{*} X\right)$ the following are equivalent:

(1) There is a fully elliptic element of the transmission algebra

$$
\mathcal{A}=\left(\begin{array}{cc}
P_{+}+G & K \\
T & Q
\end{array}\right) \in \Psi_{\text {tn }}^{0}(X ; \mathbb{E}, \mathbb{F}) \text { such that }\left[\sigma\left(P_{+}\right)\right]=p .
$$

(2) There is a fully elliptic element of the zero calculus

$$
P_{0} \in \Psi_{0}^{0}(X ; \mathbb{E}) \text { such that }\left[{ }^{0} \sigma\left(P_{0}\right)\right]=p .
$$

(3) There is a fully elliptic element of the scattering calculus

$$
P_{s c} \in \Psi_{\mathrm{sc}}^{0}(X ; \mathbb{E}) \text { such that }\left[{ }^{s c} \sigma\left(P_{s c}\right)\right]=p .
$$

(4) The Atiyah-Bott obstruction of $[p]$ vanishes, i.e.,

$$
p \in \operatorname{ker}\left(\mathrm{K}_{c}\left(T^{*} X\right) \stackrel{r^{*}}{\rightarrow} \mathrm{K}_{c}\left(T_{\partial X}^{*} X\right)\right) .
$$

Furthermore, each such realization determines a lift of the class $p$ to an element of $\mathrm{K}_{c}\left(T^{*} X, T_{\partial X} X\right)$, and the index is given by the Atiyah-Singer index theorem applied to the double of $X$.

Proof. The equivalence of (1), (2), and (3) with (4) has already been established: for (1) this is Theorem 5.14 in [6], for (2) this follows from [26, (6.12)], and for (3) this was established in Proposition 5.3 above. For each of these elements of $\mathrm{K}_{\mathrm{c}}\left(T^{*} X\right)$ there are in fact many fully elliptic elements of each calculus with this fixed symbol. This is evident for the scattering calculus, and follows for Boutet de Monvel's calculus and the zero calculus from the existence of a homotopy to an operator whose symbol is the 
identity near the boundary (the homotopy of the symbol restricted to the boundary cosphere bundle defines an extension of the symbol).

The last part of the theorem follows from a version of Proposition 2.1 in [1. Set

$$
\begin{aligned}
& Q(X, \partial X)= \\
& \quad\left\{(\alpha, \beta) \in \operatorname{ISO}_{S^{*} X}(E, F) \times \operatorname{ISO}_{B^{*} \partial X}(E, F): \alpha L_{S^{*} \partial X}=\beta \iota_{S^{*} \partial X}\right\}
\end{aligned}
$$

with distinguished subset

$$
Q^{*}(X)=\left\{\alpha \in \operatorname{ISO}_{S^{*} X}(E, F): \alpha \iota_{S^{*} \partial X}=\mathrm{Id}\right\} .
$$

Lemma 6.3. There exists a unique function

$$
Q(X, \partial X) \stackrel{f}{\rightarrow} K^{0}\left(T^{*} X, T_{\partial X}^{*} X\right)
$$

satisfying the following conditions:

i) $f(\alpha, \beta)=f\left(\alpha^{\prime}, \beta^{\prime}\right)$ if $(\alpha, \beta)$ and $\left(\alpha^{\prime}, \beta^{\prime}\right)$ are homotopic in $Q(X, \partial X)$.

ii) $f\left((\alpha, \beta) \oplus\left(\alpha^{\prime}, \beta^{\prime}\right)\right)=f(\alpha, \beta)+f\left(\alpha^{\prime}, \beta^{\prime}\right)$

iii) $f(\alpha, \operatorname{Id})=[\alpha]$ if $\alpha \in Q^{*}(X)$.

Proof. This follows directly from the analysis of the scattering case in [26]. Indeed, stable homotopy classes of elements in $Q(X, \partial X)$ are precisely the classes in $\mathcal{K}_{\mathrm{sc}}^{0}$, so the existence follows from the isomorphism

$$
\mathcal{K}_{\mathrm{sc}}^{0} \cong K^{0}\left(T^{*} X, T_{\partial X}^{*} X\right) .
$$

Uniqueness follows from an argument of Atiyah. Any $(\alpha, \beta) \in Q(X, \partial X)$ is stably homotopic to an element in $Q^{*}(X)$, i.e. , there is an element $\left(\alpha^{\prime}, \mathrm{Id}\right) \in Q^{*}(X)$ with $f\left(\alpha^{\prime}, \mathrm{Id}\right)=0$ and a homotopy of $(\alpha, \beta) \oplus\left(\alpha^{\prime}, \mathrm{Id}\right)$ to another $\left(\alpha^{\prime \prime}, \mathrm{Id}\right) \in Q^{*}(X)$, hence

$$
f(\alpha, \beta)=f\left((\alpha, \beta) \oplus\left(\alpha^{\prime}, \mathrm{Id}\right)\right)=f\left(\alpha^{\prime \prime}, \mathrm{Id}\right)=\left[\alpha^{\prime \prime}\right] .
$$

6.5. Differential operators. Given a local elliptic boundary value problem of Lopatinskii-Schapiro type, $(P, B)$, it follows from the discussion above that there is a Fredholm zero operator with the same interior symbol and index. In fact, if $P$ is a differential operator of order $k$, then $x^{k} P$ is an elliptic zero-differential operator whose interior symbol coincides with that of $P$. It is also possible to give an explicit example of a perturbation of order $-\infty$ to a fully elliptic operator corresponding to $B$.

Theorem 6.4. Let $(P, B)$ be a local, fully elliptic boundary value problem, then there exists an operator $\mathcal{B} \in \Psi_{0}^{-\infty}(X ; E, F)$ such that $x^{k} P+\mathcal{B} \in$ $\Psi_{0}^{k}(X ; E, F)$ is a fully elliptic operator with the same index as $(P, B)$. 
Proof. By assumption, $P$ is an elliptic differential operator between sections of $E$ and $F$ and $B$ is a formal differential operator at the boundary between sections of $E$ and $G$. The normal symbol of $P$ can be used to identify $E$ and $F$ near the boundary, so we can assume that $E \sim F$ near $\partial X$ and that the normal symbol of $P$ is the identity (for some choice of normal vector field). Then let $p\left(x \partial_{x}, x \widehat{\eta}\right)$ and $b\left(x \partial_{x}, x \widehat{\eta}\right)$ be the model operators obtained from the principal symbols frozen at the boundary. We shall show that the Lopatinskii-Schapiro condition is equivalent to the statement that if $\chi(x)$ is a cutoff near $x=0$ then for $\varepsilon>0$ small enough

$$
\left\langle b^{*} g, \chi^{2}\left(\frac{x}{\varepsilon}\right) b f\right\rangle
$$

is non-degenerate as a pairing between the null space of $p$ and the null space of $p^{*}$. From this it follows by continuity that the operator of order $-\infty$ in the b-calculus on $[0, \infty)$

$$
a=I_{\delta} b^{*} \chi^{2}\left(\frac{x}{\varepsilon}\right) b I_{\delta}
$$

is such that $p+a$ is invertible for small $\delta$; here $I_{\delta}$ is an approximate identity. Then $a$ is in the range of the reduced normal map and $x^{m} P+\varepsilon A$ is Fredholm for small $\varepsilon>0$ if

$$
\sigma(A)=a .
$$

To see the non-degeneracy of (6.3) observe that the Lopatinskii-Schapiro condition is the condition that

$$
\left.u \stackrel{\beta^{+}}{\longrightarrow} b\left(\partial_{x}, \widehat{\eta}\right) u\right|_{x=0}
$$

is an isomorphism from the $L^{2}$ null space of $P\left(\partial_{x}, \widehat{\eta}\right)$ to the fibre of the bundle $G$ at the corresponding point. This in turn is equivalent to the nondegeneracy of the corresponding bilinear form on the product of the null space of $p$ and of $p^{*}$

$$
\left\langle b^{*} v, b u\right\rangle_{G} .
$$

The null space of $p\left(\partial_{x}, \widehat{\eta}\right)$ is the same as that of $p\left(x \partial_{x}, x \widehat{\eta}\right)$ and the difference between (6.3) and (6.6) is that there is an integral in (6.3), cut off by $\chi$ and with additional factors of $x$. It follows that (6.3) has an asymptotic expansion as $\varepsilon \downarrow 0$ with leading term (with power determined by the orders, i.e. , the powers of $x$ ) with coefficient just (6.6). Thus, for small $\varepsilon$ the form (6.3) is indeed non-degenerate. Inserting the approximate identities as in (6.4) gives a bilinear form converging to the previous one as $\delta \rightarrow 0$ so again this is non-degenerate, now for $\delta>0$ small enough.

The resulting operator $a$ is then in the range of the reduced normal operator, e.g., its b-symbol is independent of $\widehat{\eta}$.

It follows that $\pi^{\prime} a \pi$ is an isomorphism where $\pi$ and $\pi^{\prime}$ are the projections onto the null space of $p$ and that of $p^{*}$. An element in the null space of the 
operator $p+\varepsilon a$ can be decomposed according to these projections, into $u+v$ with $u$ in the null space of $p$ and $v$ orthogonal to it. Then it must satisfy

$$
\left(p+\epsilon a_{11}\right) u_{1}+\epsilon a_{12} v=0, a_{21} u+a_{22} v=0
$$

where $a_{i j}$ is the $2 \times 2$ decomposition of $a$ with respect to these projections. By arrangement, $a_{22}$ is invertible so this reduces to

$$
\left(p+\varepsilon\left(a_{11}-a_{12} a_{22}^{-1} a_{21}\right)\right) u=0 .
$$

For $\varepsilon>0$ this has no solutions, by the invertibility of $p$ as a map from the orthocomplement of its null space to its range. Thus the reduced normal operator is invertible and hence the operator is $P+\epsilon A$ Fredholm.

Finally, recall from [1] that the isomorphism $\beta^{+}$, hence the quadratic form (6.6), determines the index and indeed the lift of the K-class of $\sigma(P)$ from $K\left(T^{*} X\right)$ to $K\left(T^{*} X, T^{*} \partial X\right)$. Thus the index of $x^{k} P+\mathcal{B}$ is the same as that of $(P, B)$.

\section{REFERENCES}

[1] M. F. Atiyah, The index theorem for manifolds with boundary, Seminar on the AtiyahSinger index theorem, Princeton University Press, Princeton, N.J., 1965, pp. 337-351.

[2] M. F. Atiyah, K-theory. Notes by D. W. Anderson. Second edition. Advanced Book Classics. Addison-Wesley Publishing Company, Advanced Book Program, Redwood City, CA, 1989. xx+216.

[3] M. F. Atiyah and R. Bott, The index problem for manifolds with boundary, Differential Analysis, Bombay Colloq., 1964, Oxford Univ. Press, London, 1964, pp. 175-186.

[4] B. Booss and D. D. Bleecker, Topology and analysis, Universitext, Springer-Verlag, New York, 1985, The Atiyah-Singer index formula and gauge-theoretic physics, Translated from the German by Bleecker and A. Mader.

[5] Bernhelm Booß-Bavnbek and Krzysztof P. Wojciechowski, Elliptic boundary problems for Dirac operators, Mathematics: Theory \& Applications, Birkhäuser Boston Inc., Boston, MA, 1993.

[6] Louis Boutet de Monvel, Boundary problems for pseudo-differential operators, Acta Math. 126 (1971), no. 1-2, 11-51.

[7] Jochen Ditsche, K-theory for pseudodifferential operators on conformally compact spaces, Preprint 04/2007, available online at ww.mathematik.unimainz.de/Aktuelles/vorabdrucke/Ditsche.pdf

[8] Charles L. Epstein, Richard B. Melrose, and Gerardo A. Mendoza, Resolvent of the Laplacian on strictly pseudoconvex domains., Acta Math. 167 (1991), no. 1-2, 1-106.

[9] Andrew Hassell, Rafe Mazzeo, and Richard B. Melrose, Analytic surgery and the accumulation of eigenvalues, Comm. Anal. Geom. 3 (1995), no. 1-2, 115-222.

[10] Lars Hörmander, Linear partial differential operators, Die Grundlehren der mathematischen Wissenschaften, Bd. 116, Academic Press Inc., Publishers, New York, 1963.

[11] H. Inassaridze and T. Kandelaki1, Smooth K-theory of locally convex algebras, Arxiv 0603095, March 2006.

[12] Robert Lauter, Pseudodifferential analysis on conformally compact spaces., Mem. Amer. Math. Soc. 163 (2003), no. 777, xvi+92pp.

[13] Paul Loya, Dirac operators, boundary value problems, and the b-calculus, Spectral geometry of manifolds with boundary and decomposition of manifolds, Contemp. Math., vol. 366, Amer. Math. Soc., Providence, RI, 2005, pp. 241-280. 
[14] Paul Loya and Richard B. Melrose, Fredholm perturbations of Dirac operators on manifolds with corners, preprint, 2003.

[15] Rafe Mazzeo, The Hodge cohomology of a conformally compact metric, J. Differential Geom. 28 (1988), no. 2, 309-339.

[16] _ Elliptic theory of differential edge operators. I, Comm. Partial Differential Equations 16 (1991), no. 10, 1615-1664.

[17] Rafe Mazzeo and Richard B. Melrose, Meromorphic extension of the resolvent on complete spaces with asymptotically constant negative curvature, J. Funct. Anal. 75 (1987), no. 2, 260-310.

[18] , Pseudodifferential operators on manifolds with fibred boundaries, Asian J. Math. 2 (1998), no. 4, 833-866, Mikio Sato: a great Japanese mathematician of the twentieth century.

[19] Severino T. Melo, Ryszard Nest, and Elmar Schrohe, K-theory of Boutet de Monvel's algebra, Noncommutative geometry and quantum groups (Warsaw, 2001), Banach Center Publ., vol. 61, Polish Acad. Sci., Warsaw, 2003, pp. 149-156.

[20] Severino T. Melo, Thomas Schick, and Elmar Schrohe, A K-theoretic proof of Boutet de Monvel's index theorem for boundary value problems., arXiv:math.KT/0403059, March 2004.

[21] Richard B. Melrose, The Atiyah-Patodi-Singer index theorem, Research Notes in Mathematics, vol. 4, A K Peters Ltd., Wellesley, MA, 1993.

[22] Richard B. Melrose and Victor Nistor, Homology of pseudodifferential operators $i$. manifolds with boundary., arXiv:funct-an/9606005. June 1996.

[23],$K$-theory of $C^{*}$-algebras of b-pseudodifferential operators, Geom. Funct. Anal. 8 (1998), no. 1, 88-122.

[24] Richard B. Melrose and Paolo Piazza, An index theorem for families of Dirac operators on odd-dimensional manifolds with boundary, J. Differential Geom. 46 (1997), no. 2, 287-334.

[25] Richard B. Melrose and Frédéric Rochon, Families index for pseudodifferential operators on manifolds with boundary, Int. Math. Res. Not. (2004), no. 22, 1115-1141.

[26] , Index in K-theory for families of fibred cusp operators., arXiv:math.DG/0507590, July 2005.

[27] Frédéric Rochon, Bott periodicity for fibred cusp operators., arXiv:math.DG/0408225, August 2004.

[28] Anton Savin, Elliptic operators on manifolds with singularities and K-homology., KTheory 34 (2005), no. 1, 71-98.

[29] Bert W. Schulze, An algebra of boundary value problems not requiring ShapiroLopatinskij conditions., J. Funct. Anal. 179 (2001), no. 2, 374-408.

[30] Bert W. Schulze and Jörg Seiler, Boundary value problems with global projection conditions., J. Funct. Anal. 206 (2004), no. 2, 449-498.

[31] Bert W. Schulze and Jörg Seiler, Edge operators with conditions of Toepltiz type., J. Inst. Math. Jussieu 5 (2006), no. 1, 101-123.

Department of Mathematics, Massachusetts Institute of Technology

E-mail address: pierre@math.mit.edu

E-mail address: rbm@math.mit.edu 